\title{
Editorial
}

\section{Deep Learning in Mobile Information Systems}

\author{
Malik Jahan Khan $\mathbb{D}^{1},{ }^{1}$ Irfan Awan $\mathbb{D}^{2},{ }^{2}$ and Zeeshan Ali Rana $\mathbb{D}^{3}$ \\ ${ }^{1}$ Department of Computer Science, Namal Institute, Mianwali, Pakistan \\ ${ }^{2}$ Faculty of Engineering and Informatics, University of Bradford, Bradford, UK \\ ${ }^{3}$ Department of Computer Science, NUCES-FAST, Lahore, Pakistan \\ Correspondence should be addressed to Malik Jahan Khan; malik.jahan@namal.edu.pk
}

Received 17 June 2020; Accepted 17 June 2020; Published 27 January 2021

Copyright (c) 2021 Malik Jahan Khan et al. This is an open access article distributed under the Creative Commons Attribution License, which permits unrestricted use, distribution, and reproduction in any medium, provided the original work is properly cited.

The usage and dependence of mobile computing devices as well as mobile information systems have exponentially increased in the recent past. The adaptation of modern technologies in the developed world has completely redesigned the canvass of the daily life of a common person. Even in the developing world, it has penetrated and is still penetrating at a very fast pace. Such systems and applications now encompass a broad range of application domains in today's world including, but not limited to, healthcare, education, e-commerce, agriculture, forestry, weather, livestock, security, and social networking. Most of the applications address the problems of identification, recognition, diagnosis, predictions, reasoning, interpretation, and summarizations. Computer vision and machine learning have intersected decently to effectively cover these application as well as problem domains.

In the same era, machine learning has evolved as a panoramic science for almost all disciplines of computer science, with intelligence being embedded at the core of information systems. Theory and applications of supervised, unsupervised, and reinforcement learning have significantly been improved in the recent years. In most of the machine learning paradigms, theories of learning and cognitive sciences have contributed as base lines. Neural networks are one of such paradigms. The fundamental building block of a neural network is a neuron shown in Figure 1. One neuron takes inputs from its universe of discourse, aggregates the input while assigning a weight to each input, and passes the output through a transfer function and a final output is generated.
In conventional supervised learning, the generated output is compared with the target output provided by the trainer and an error is computed using the following equation:

$$
E=o_{j}-t_{j}
$$

where $o_{j}$ is the output of the neuron and $t_{j}$ is the target output.

The error propagates back to compute the rate of change of error with respect to each weight using chain rule depicted in the following equation:

$$
\frac{\partial E}{\partial w_{i j}}=\frac{\partial E}{\partial o_{j}} \frac{\partial o_{j}}{\partial w_{i j}}=\frac{\partial E}{\partial o_{j}} \frac{\partial o_{j}}{\partial \text { net }_{j}} \frac{\partial \text { net }_{j}}{\partial w_{i j}} .
$$

The step size of this differential is controlled through the learning rate and change in each weight is computed using the following equation:

$$
\Delta w_{i j}=-\eta \frac{\partial E}{\partial w_{i j}}
$$

where $\Delta w_{i j}$ is the change in the weight $w_{i j}$ and $\eta$ is the learning rate. Each weight is updated using the update rule given in equation (4) and the process is repeated with the expectation that the new weights would have gotten closer to the target weights.

$$
w_{i j}^{\prime}=w_{i j}+\Delta w_{i j},
$$

where $w_{i j}^{\prime}$ is the updated weight. It is applied across all edges of the neural network. 


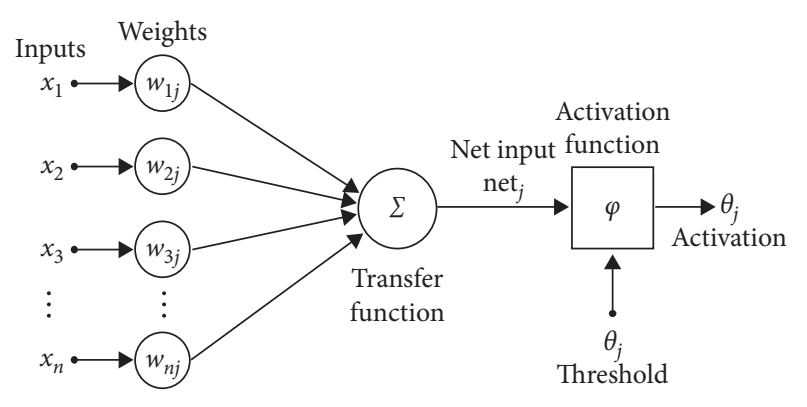

Figure 1: Building block of a neural network.

This back-propagation procedure is repeated across multiple iterations depending on the complexity of the target function. Different neurons are put together to generate a neural network to address the complex problems which may not be solvable by single neuron. Different neurons are arranged in different layers. Depth of the neural network is primarily determined by the number of hidden layers. The deeper neural networks are capable to learn the complex problem domains with the compromise on the computational complexity. Deep learning has successfully enhanced the effectiveness of mobile information systems in recent years to serve many different purposes, including object recognition, fault diagnosis, health monitoring, malware detection, and language translation. Over the last decade, mobile information systems have become more robust, autonomous, and self-organized, making tasks performed through these systems more reliable. Deep learning-based algorithms, models, and techniques, such as convolutional neural networks, probabilistic gradient algorithms, adaptive subgradient methods, and distributed deep learning over cloud methods, have been proposed, implemented, and deployed as the core decision-making engines in these systems.

The aim of this special issue of mobile information systems is to highlight the recent innovations where deep learning has been exploited to enhance the effectiveness of mobile applications and computing devices. This special issue includes papers that report innovative applications of deep learning for tasks such as visual analysis and classification in different fields including geology, indoor localization, animal species recognition, and human-computer interaction. This issue also includes a survey paper that summarizes typical applications of computational-resourcelimited deep learning and presents a list of challenges to be addressed.

Fan et al. have presented a deep learning model for quick and accurate rock recognition with smart phones to help geological surveys. Recognition and classification of rock lithology is an important topic in geographical sciences. A lightweight convolutional neural network (CNN) has been trained to correctly recognize and classify rock images.

Ji et al. have proposed an indoor classification mechanism based on multiple descriptors fusion. Descriptor filter algorithms using a greedy approach have been proposed and implemented. Its performance has been analyzed and simulation results have been presented.
Choe et al. have presented a CNN to classify the endangered parrot species. The proposed approach has been deployed with a real-time mobile application. The application is quite innovative and significant to protect the endangered species of parrot. It has significant application to prevent smuggling and assist the relevant authorities identify breaches.

Liu et al. have presented a deep learning algorithm to recognize hand gestures. Hand gestures in complex scenarios have been chosen to be tested using single shot multibox detector deep learning algorithm. The proposed setup has been tested in real-time situations and high classification accuracy has been achieved. This adds significant value from the human-computer interaction perspective.

Wang et al. have presented a modification in MobileNet (a lightweight convolutional neural network) to improve its efficiency and capacity to integrate with mobile platforms. Image classification has been successfully tested in the implementation and analysis of the proposed framework.

Chen et al. have presented a survey on the deployment of deep learning in computational-resource-limited platforms. Deep learning is quite expensive in terms of resource requirements due to its inherent multilayer architecture and huge number of iterations required to execute the gradientdescent algorithm. The topic addressed in this survey paper is worth exploring to maintain a balance between the two extremes: computational expense and available resourcelimited platforms.

With the intervention of sophisticated computational resources globally available through cloud computing, deep learning applications have been extensively developed and deployed. Conventional machine learning has been largely evolved into artificial neural networks and deep learningbased models primarily due to their potential to solve complex problems. With the advent of smarter computational resources for mobile devices, deep learning has immense potential to enable and embed intelligence in all kinds of applications and systems running over mobile devices.

\section{Conflicts of Interest}

The editors declare that there are no conflicts of interest regarding the publication of this special issue.

Malik Jahan Khan Irfan Awan Zeeshan Ali Rana 